\title{
Comparison Theorems for Conjugate Points of Sturm-Liouville Differential Equations
}

\author{
E. MÜLLER-Pfeiffer
}

Es werden Vergleichssätzè für Lösungen Sturm-Liouvillescher Differentialgleichungen bezüg.

- lich ihrer Nullstellen auf einem endlichen Intervall bewiesen.

Доказываются теоремы сравнения решения "дифференциајьных уравнениі ШтурмаЛиувиля относительно пх нулей иа конечном интервале.

Comparison theorems for solutions of Sturm-Liouville equations are proved concerning their zeros on a finite interval.

Consider the differential equations

and

$$
-\left(P(x) u^{\prime}\right)^{\prime}+Q(x) u=0, \quad-a \leqq x \leqq \varliminf^{\prime} u,
$$

$$
: \quad-\left(p(x) u^{\prime}\right)^{\prime}+q(x) u=0, \quad-u \leqq x \leqq \iota,
$$

$a \in \mathbf{H}$, where the coefficients are real-valued continuous functions and, additionally, $p, P$ are positive piecewise and continuously differentiable. The points $x_{1}, x_{2} \in[-a, a]$ are 'said to be conjugate points for equation (1) or (2) if there exists a nontrivial solution $u$ of the corresponding equation with $u\left(x_{1}\right)=0=u\left(x_{2}\right)$. We prove the following

Thèrem 1: Assume the following:

(i) $P$ and $Q$ are even functions on $[-a, a]$,

(ii) $p \leqq P$ on $[-a, u]$,

(iii) $\int_{[-\sigma, a]}^{\prime} q d x \leqq \int_{[-\sigma, \sigma] \backslash(-\tau, \tau)} Q d x$ for all $\sigma, \tau$ with $0<\tau<\sigma<a$.

If there exists ' $a$ solution $u$ of (1) with $u(-a)=0=u(a)$ and $u(x)>0,-a<x<a$, then there exists a pair of conjugate points for equation (2) on $[-a, a]$.

Proof: The solution $u$ of (1) belongs to $C^{\mu}[-a, a]$ (cp. [.7: p. 25], for instance). By using the Sturm conparison theorem (cp. [7]) it is easily.seen that such $u$ is an even function. By means of $u$ we construct a test function for the quadratic form of equation (2). Let $\alpha \in[0, a)$ be a point with $u^{\prime}(\alpha)=0$ and $u^{\prime} \leqq 0$ on $[\alpha, a]$. (Note that there can exist several points $\alpha$ with this property.) It is easily seen that the function

$$
w(x)= \begin{cases}u(\alpha), & x \in(-\alpha, \alpha) \\ u(x), & x \in[-\alpha, a] \backslash(-\alpha, \alpha)\end{cases}
$$


belongs to the Sobolev spaces $\dot{W}_{2}^{1}(-a, a)$ and $\left.W_{2}^{2}(-a, a)^{1}\right)$. The sequilinear forms of the equations (1) and (2) defined on $W_{2}^{1}(-a, a)$ are closed. In the following, by means of $w$, the quadratic form of (2) will be estimated. Thus, by using Fubini's theorem, the function.

$$
h(y):=\sup \left\{x \in[0, u] \mid w^{2}(x) \geqq y\right\}, \quad 0 \leqq y \leqq u^{2}(\alpha),
$$

and the hypotheses (ii) and (iii), we obtain

$$
\begin{aligned}
& \int_{-a}^{a}\left[p\left(w^{\prime}\right)^{2}+q w^{2}\right] d x=\int_{-a}^{a}\left[(p-P)\left(w^{\prime}\right)^{2}+(q-Q) w^{2}\right] d x+\int_{-a}^{a}\left[P\left(w^{\prime}\right)^{2}+Q w^{2}\right] d x \\
& \leqq \int_{-a}^{a}(q-Q) w^{2} d x+\left.P u^{\prime} u\right|_{-a} ^{-\alpha^{\prime}}+\int_{-a}^{-\infty}\left[-\left(P u^{\prime}\right)^{\prime}+Q u\right] u d x \\
& +u^{2}(\alpha) \int_{-\alpha}^{a} Q d x+\left.P u^{\prime} u\right|_{a} ^{a}+\int_{a}^{a}\left[-\left(P u^{\prime}\right)^{\prime}+Q u \cdot\right] u d x \\
& =\int_{-a}^{a}(q-\dot{Q}) w^{2} d x+u^{2}(\alpha) \int_{-\infty}^{a} Q d x \\
& =\int_{-a}^{a} \int_{0}^{w^{2}(x)}[Q(x)-Q(x)] d y d x+u^{2}(\alpha) \int_{-\alpha}^{a} Q(x) d x \\
& =\int_{0}^{u^{z_{(}(x)}}\left(\int_{-h(y)}^{h(y)}[q(x)-Q(x)] d x+\int_{-\alpha}^{\alpha} Q(x) d x\right) d y \\
& \stackrel{u^{u^{2}(\alpha)}}{=} \int_{0}\left(\int_{[-h(y) \cdot h(y)]} q(x) \cdot d x-\int_{1-h(y) \cdot h(y))(-\alpha, 2)} Q(x) d x\right) d y \leqq 0 .
\end{aligned}
$$

Therefore,

$$
\inf \left\{\int_{-a}^{a}\left(p\left|\varphi^{\prime}\right|^{2}+q|\varphi|^{2}\right) d x: \varphi \in C_{0}^{\infty},\|\varphi\|=1\right\}
$$

is either less or equal zero.

In the first case it follows that there exists a nontrivial solution $v$ of (2) having at least two zeros on $(-a, a)$. (cp. [5]). In the second case the (normalized) function $w$ is realizing the infimum, and, consequently, it is a solution of (2). This can be proved as follows. Let $A$ be the Friedrichs extension of the operator

$$
\dot{A}_{0} \varphi=-\left(p \varphi^{\prime}\right)^{\prime}+q \varphi, \quad \varphi \in C_{0}^{\infty}(-a, a) \text {. }
$$

- Because of $\left.\left(A_{0} \varphi, \varphi\right) \geqq 0^{2}\right), \varphi \in C_{0}^{\infty}(-u, a)$, the operator $A^{1 / 2}, D\left(A^{1 / 2}\right)=\hat{W}_{2}{ }^{1}(-u, a)$, - can be defined (cp. [3]). Then, it follows from

$$
0=\int_{-a}^{a}\left[p\left(w^{\prime}\right)^{2}+q w^{2}\right] d x=\left\|A^{1 / 2} w\right\|^{2}
$$

1) $\mathrm{IF}_{2} \mathbf{i}(-a ; a)$ is the completion of $C_{0}^{\infty}(-a, a)$ in the $w_{2}^{\prime}-$ norm.

$\Rightarrow(\cdot, \cdot)$ and $\|\cdot\|$ denote inner product and norm in the Hilbert space $L,(-a, a)$, respectively. 
that $A^{1 / 2} w=0$ and $0=A^{1 / 2}\left(A^{i / 2} w\right)=A w$. By the first representation theorem (see [3: p. 322]) we have

$$
\int_{-a}^{a}\left(\eta u^{\prime} \bar{v}^{\prime}+q u \bar{v}\right) d x=(A w, v)=0 \text { for every } v \in \hat{W}_{2}^{1}(-a, a),
$$

and by integration by parts it follows that

$$
\int_{-a}^{a}\left[\div\left(p w v^{\prime}\right)^{\prime}+q u\right] \bar{v} d x=0 \text { for all } v \in \dot{W}_{2}{ }^{1}(-a, a) \text {. }
$$

Hence, we obtain $-\left(\dot{p} w^{\prime}\right)^{\prime}+q w=0$. The solution $w$ of (2) has zeros at the end points of the interval $[-u, a]$.

We state that in both cases there exists.a pair of conjugate points for equation (2) on $[-a, a]$. This completes the proof of Theorem 1

If a point $\alpha \in[0, a)$ with the named properties is known, Theorem 1 can be modified as follows.

Corollary 1: Assume the following:

(i) $P$ and $Q$ are even functions on $[-a, a]$,

(ii) $p \leqq P$ on $[-a, a]$.

Assume that there exists a solution $u$, of (1) with $u(-a)=0=u(a) ; u(x)>0$, $-a<x<a ; u^{\prime}(\alpha)=0$ and $u^{\prime} \leqq 0$ on $[\alpha, a]$. If, additionally,

$$
\int_{1-\sigma, \sigma]} q d x \leqq \int_{1-\sigma, \sigma)(-\alpha, \alpha)} Q d x \text { for } a l l \sigma \in(\alpha, a)
$$

then there exists a pair of conjugate points for equation (2) on $[-a, a]$.

Proof: Compare the proof of Theorem 1

In the special case that

$$
\int_{0}^{0} Q d x \leqq 0 \text { when } \sigma \in(0, a)
$$

the solution $u$ is monotone decreasing on $[0, a]$. This can be seen as follows. By setting $v=-P u^{\prime} u^{-1}$ it. follows from $u v^{\prime}+u^{\prime} v=(u v)^{\prime}=-\left(P u^{\prime}\right)^{\prime}=-Q u$ that $v$ satisfies the Riccati differential equation $v^{\prime}=-Q+P^{-1} v^{2}$. Because of $u^{\prime}(0)=0$ we have $v(0)=0$ and $v^{\prime} \equiv-Q^{\prime}+P^{-1} v^{2}$ implies

$$
v(x)=-\int_{0}^{x} Q d t+\int_{0}^{x} P-1 v^{2} d t, \quad 0 \leqq x \leqq u .
$$

In view of (3) and (4) we obtain $v \geqq 0$ and, consequently, $u^{\prime} \leqq$ on $[0, u]$ : Therefore, by assuming (3), the point $\alpha$ can be chosen equal to zero. Thus; we obtain the following result from Corollary 1.

Corollary 2: Assume the following:

(i) $P$ and $Q$ are even functions on [-a, $a]$,

\}. (ii) $p \leqq P$ on $[-a, a]$,

(iii) $\int_{-\sigma} q d x \leqq \int_{-5} Q d x \leqq 0$ for all $\sigma \in(0, a)$. 
If there exists a solution $u$ of (1) with $u(-u)=0=u(a)$ and $u(x)>0,-u<x<a$, then there exists a pair of conjugate points for equation (2) on [-a, a].

A similar result of Corollary 2 was obtained by Fisk [2: Th. 2] under the hypothesis $p \equiv P$ (cp. [7: p. 186]). In the special case $p \equiv P \equiv 1$ and $Q \leqq 0$ Corollary 2 is a result of LeightoN [4: Th. 1.3]. ing:,

Corollary 3: Consider the equations (1) and (2) on [0, a] and assume the follow-

(i) $p \leqq P$ on $[0, a]$,

(ii) $\int_{0}^{\sigma} q d x \leqq \int_{\mathrm{r}}^{\sigma} Q d x$ for all $\sigma, \tau$ with $0<\tau<\sigma<a$

or

(ii') $\int_{0}^{\sigma} q d x \leqq \int_{0}^{o} Q d x \leqq 0$ jor all $\sigma \in(0, a)$.

If there exists a solution $u$ of $(1)$ on $[0, a]$ with $u^{\prime}(0)=u(a)=0$ and $u(x)>0,0 \leqq x$ $<a$, then every solution $v$ of $(2)$ on $[0, a]$ with $v(0)>0$ and $v^{\prime}(0) \leqq 0$ hus a zero on. $(0 ; a]$.

Proof: By using the Sturm comparison theorem it is easily seen that it is sufficient to prove Corollary 3 under the hypothesis $v^{\prime}(0)=0$ in place of $v^{\prime}(0) \leqq 0$. Then, extend the functions $p, P, q, Q$, and the solutions $u$ and $v$ as even functions on $[-a, a]$ and use Theorem 1 (or Corollary 2) with the aid of Sturm's comparison. theorem

In the following the hypothesis $p \leqq P$ is to be weakened. Henceforth, let $P$ be continuously differentiable on $(0, a]$..

Theorem 2: Assume the following:

(i) $P$ and $Q$ are even functions on $[-a, a]$,

(ii) $Q, P^{\prime} \leqq 0$ on $(0, a]$,

(iii) $\int_{I-a, a] \backslash(-\sigma, \sigma)}[P, p] d x \geqq 0$ and $\int_{-\sigma}^{\sigma}[Q-q] d x \geqq 0$ jor all $\sigma, 0<\sigma<a$.

If there exists a solution $u$ of (1) with $u(-a)=0=u(a)^{\prime}$ and $u(x)>0,-i<x<a$, then there exists a pair of conjugate points for equatioin (2) on $[-a, a]$.

- Proof: $Q \leqq 0$ implies inequality (3). Therefore, as shown in the proof of Corollary 1 , we have $u^{\prime} \leqq 0$ on $[0, a]$ and by means of $P^{\prime} \leqq 0$ it follows from $P u^{\prime \prime} \equiv-P^{\prime} u^{\prime}$ $+Q u$ on $(0, a]$, that $u^{\prime \prime} \leqq 0$ on $(0,-a]$. Consequently, the derivative $u^{\prime}$ is monotone decreasing on $[0, a]$. 'The function $\left(u^{\prime}\right)^{2}$ is even and monotone increasing on $[0, a]$. Hence, by means of the hypothesis (iii) we obtain

$$
\begin{aligned}
& \int_{-a}^{a}(p-P)\left(u^{\prime}\right)^{2} d x=\int_{-a}^{a} \int_{0}^{a\left(u^{\prime}(x)\right)^{2}}[p(x)-P(x)] d y d x \\
& =\int_{0}^{\left[u^{\prime}(a)\right]^{2}} \int_{[-a, a] \backslash(-h(y), h(y))}[p(x)-P(x)] d x d y \leqq 0
\end{aligned}
$$


where $h(y)=\sup .\left\{x \in[0, a] \mid\left[u^{\prime}(\dot{x})\right]^{2} \leqq y\right\}, 0 \leqq y \leqq\left[u^{\prime}(a)\right]^{2}$. Analogously (compare the proof of Theorem 1), we get $\int_{-a}^{a}(q-Q) u^{2} d x \leqq 0$. Thus, it follows that

$$
\int_{-a}^{a}\left[p\left(u^{\prime}\right)^{2}+q u^{2}\right] d x=\int_{-a}^{a}\left[(p-P)\left(u^{\prime}\right)^{2}+(q-Q) u^{2}\right] d x \leqq 0 .
$$

Finally, finish the proof as the proof of Theorem $1^{\circ}$

Corollary 4: Consider the equations (1) and (2) on $[0, a]$ and assume the following:

(i) $Q, P^{\prime} \leqq 0^{\circ}$ on $[0, a]$,

(ii) $\int_{0}^{a} P d x \geqq \int_{0}^{a} p d x$ and $\int_{0}^{a} Q d x \geqq \int_{0}^{a} q d x$ for all $\sigma \in(0, a)$.

If there exists a solution $u$ of (1) with $u^{\prime}(0)=0=u(a){ }^{\prime}$ and $u(x)>0,0 \leqq x<u$, then every solution of $(2)$ with $v(0)>0$ and $\dot{v}^{\prime}(0) \leqq 0$ has a zeró on $(0, a]$.

Proof: Compare the proof of Corollary 3

Corollaries 3 and 4 are generalizations of theorems of NeHARI [6] and I.EIGHTON [4: Th. 1.1].

Corollary 5: If the inequality

$$
\sup _{\sigma \in(0, a)} \frac{1}{\sigma} \int_{0}^{\sigma} q d x \leqq-\frac{\pi^{2}}{4 a^{2}} \sup _{\sigma \in(0, a)} \frac{1}{a-\sigma} \int_{0}^{a} p d x
$$

holds, then every solution $v$ of $(2)$ on $[0, a]$ with $x(0)>0$ and $v^{\prime}(0) \leqq 0$ has a zero. on $(0, a]$.

Proof: Define

$$
\varrho=\sup _{\sigma \in(0, a)} \frac{1}{a-\sigma} \int_{a}^{a} p d x
$$

and set $P \equiv \varrho$ and $Q \equiv-\pi^{2} \varrho / 4 a^{2}$. The function $u=\cos (\pi x / 2 a)$ is a solution of the differential equation

$$
\text { 1. } \varrho u^{\prime \prime}-\frac{\pi^{2} \varrho}{4 a^{2}} u=0, \quad 0 \leqq x \leqq a,
$$

with the properties supposed in Corollary 4 . Hypothesis (i) of Corollary 4 is fulfilled. It follows from (5) and (6) that (ii) also holds. This proves Corollary 5

Example: Consider the differential equation

Since

$$
-\left(\left(1-x^{2}\right) u^{\prime}\right)^{\prime}-6 u=0, \quad 0 \leqq x \leqq a<1 .
$$

$$
\frac{1}{a-\sigma} \int_{\sigma}^{a}\left(1-x^{2}\right) d x \leqq \frac{1}{a} \int_{0}^{a}\left(1-x^{2}\right) d x=1-\frac{a^{2}}{3}, \quad \sigma \in(0, a),
$$


we have $\varrho=1^{1}-a^{2} / 3$. Inequality (5) holds when $a \geqq a_{1}=\left(3 \pi^{2} /\left(72+\pi^{2}\right)\right)^{1 / 2} \approx 0,6014$. Hence, every solution $v$ of $(7)$ with $v(0)>0^{\circ}$ and $v^{\prime}(0) \leqq 0$ has a zero on $\left(0, a_{1}\right]$. Because $v=1-3 x^{2}$ is a solution of $(7)^{\prime}$ with $v^{\prime}(0)=0$, the smallest $a$ is $a_{0}=3^{-1 / 2} \approx 0.5774$. We state that $a_{1}$ is a good approximate value for. $a_{0}$.

\section{REFERENCES}

[1] Browder, F. E.: On the spectral theory of elliptic differential operators I. Math. Ann. 142 (1961), 22-130.

[2]-Fink, A. M.: Comparison theorems for eigenvalues. Quart. Appl. Math. 2S (1970), 289-292.

[3] Kato, T.: Perturbation theory for linear operators. Berlin-Heidelberg-New York: Springer-Verlag 1966.

[4] Leigiton, W.:'Some oscillation theory. Z. Angew. Nath. Mech. 63 (1983), 303, 315.

[5] MÜlLER-PFEIFFER, E.: On the existence of nodal domains for elliptic differential operators. Proc. Roy. Soc. Edinburgh 94 A (1983), $287-299$.

[6]. Nefari, Z.: Oscillation criteria for second-order linear differential equations. 'Trans. Amer. Math. Soc. 85 (1957), $428-445$.

[7] ReID, W. T. : Sturmian theory for ordinary differential equations (Applied Nathematical Sciences, Vol. 31). New York-Heidelberg-Berlin: Springer-Verlag 1980.

Manuskripteingang: $10.05 .198 . \overline{5}$

\section{VFRFASSER:}

Prof. Dr. ERich Müllek-PFEIfFer

Sektion Mathematik/Physik

der Pädagogișchen Hochschule ,.Dr. Th. Neubauer"

DDR - 5010 Erfurt, Nordhäuser Str. 63 